\title{
On the material dependence of experimental shear fracture orientation
}

\author{
Enrique Gomez-Rivas $^{1 *}$ and Albert Griera ${ }^{2}$ \\ ${ }^{1}$ Department of Geology and Petroleum Geology, School of Geosciences, King's College, University of \\ Aberdeen, Aberdeen AB24 3UE, UK; \\ ${ }^{2}$ Departament de Geologia, Universitat Autònoma de Barcelona, 08193 Bellaterra (Cerdanyola del Vallès), \\ Spain
}

Received March 7, 2015; accepted October 30, 2015; first online December 3, 2015

\begin{abstract}
It is not uncommon to observe shear fractures in ductile rocks oriented at more than $45^{\circ}$ with respect to the maximum compression direction. Since these orientations cannot be explained with the classic Mohr-Coulumb or Tresca yield criteria, Zheng et al. (Journal of Structural Geology, 35: 1394-1405, 2011) proposed the maximum effective moment (MEM) failure criterion. This rule suggests that shear fractures in ductile rocks form at $\sim 55^{\circ}$ with the maximum compression axis and that this orientation is material-independent and, therefore, universal. Zheng et al. (Science China: Earth Sciences, 57(11): 2819$2824,2014)$ used data from our own experiments as supporting evidence of their failure criterion. In this contribution we discuss why shear fracture formation in ductile rocks indeed strongly depends on the mechanical properties of the deforming medium, and why experimental data should not be taken to prove the validity of the MEM criterion. The formation mechanisms and orientations of shear fractures in our experiments significantly vary depending on the material strength and degree and type of anisotropy (composite and intrinsic). We therefore demonstrate using experimental data that a universal failure angle in ductile and anisotropic rocks does not apply. Additionally, we highlight some inconsistencies of the MEM criterion.
\end{abstract}

Keywords: shear fracture, anisotropy, fracture orientation, material properties, failure criteria, ductile deformation

This manuscript published in the journal Science China Earth Sciences (2015), 58(12), 2357-2362.

The present document is an author's (post-print) version. For the final copyedited version, please visit http://link.springer.com/article/10.1007/s11430-015-5217-0?wt_mc=internal.event.1.SEM.ArticleAuthorOnl ineFirst

DOI: $10.1007 / \mathrm{s} 11430-015-5217-0$

Failure criteria are used to predict the conditions under which solid materials, such as rocks, fail when they are subjected to an external load. The onset and orientation of shear fractures in isotropic rocks is typically predicted with the Mohr-Coulomb failure criterion, which is represented by an envelope in a plot of material shear strength against applied normal stress (e.g. Jaeger and Cook, 1979). This criterion considers that the angle between the shear fracture plane and the principal compressive stress $\left(\sigma_{1}\right)$ actually depends on the material as well as on the stress state (and consequently on the confining pressure), since the failure envelope in shear stress vs normal stress space is a material property. This angle becomes higher with increasing confining pressure, and can reach up to $45^{\circ}$ when the rock starts to behave plastically. Fractures can form at this orientation once the plastic yield stress has been reached, and then follow the Tresca, or maximum shear stress orientation, yield criterion (Twiss and Moores, 1992). At this point yielding becomes independent of the differential stress. A fundamental problem of these criteria is that they are unable to predict fractures oriented at more than $45^{\circ}$ with respect to $\sigma_{1}$, such as for example low-angle normal faults and high-angle reverse faults (see Zheng et al., 2011 and Gomez- Rivas and Griera, 2012 and 
references therein). These types of fractures are not uncommon in isotropic or anisotropic rocks that are deformed in dominant ductile conditions, and therefore a failure criterion to describe their onset is lacking. For that reason, Zheng et al. (2004, 2011, 2012) proposed the "maximum-effective-moment" (MEM) failure criterion. The main implication of this criterion is that the angle between shear fractures and the maximum compression stress $\left(\sigma_{1}\right)$ has a constant value of $55^{\circ}$, and it is material-independent.

Recently, Zheng et al. (2014) have published in this journal a manuscript titled "Interpretation of the experimental data provided by Gomez-Rivas and Griera (2012) in terms of the MEM-criterion”. These authors used the experimental data published in Gomez-Rivas and Griera (2012) to support their failure criterion, because the angles at which shear fractures nucleate in these experiments fit their predictions. Their contribution does not criticise or question our experimental results, but attempts to provide an alternative interpretation of our models. One of the main findings of the experiments published in Gomez-Rivas and Griera (2012) is the development of large angles between conjugate shear fracture sets and $\sigma_{1}$ in anisotropic materials. For simplicity, here we use the term shear fracture to refer to both shear fractures and shear bands. In this contribution, we refrain from discussing the theory underlying the definition of the MEM criterion (see Tong, 2012), but instead we prove that our experiments should not be taken as supporting evidence for the MEM theory. The $\sim 55^{\circ}$ average angle between shear fractures and $\sigma_{1}$ in our models is a mere coincidence for the specific configuration, material and strain rate we used. The attempt of applying the MEM criterion to experimental data points out some of its inconsistencies, which we briefly discuss. We show that the orientation of shear fractures actually depends on the mechanical properties of the deforming materials, and therefore a material-independent criterion is not applicable.

\section{Fracture formation angles and nucleation mechanisms}

The results of Gomez-Rivas and Griera (2012) summarise part of the experiments presented in the $\mathrm{PhD}$ thesis of Gomez-Rivas (2008), which was also taken by Zheng et al. (2011) as supporting evidence for their criterion. This thesis reports three series of experiments of coaxially-deformed anisotropic plasticine multilayers, among other field and numerical studies:

(1) A series using a mixture of OCLU-PLAST plasticine, vaseline and paper flakes deformed at the same strain rate, but systematically varying the layering/anisotropy orientation with respect to the extension axis $X$ from $0^{\circ}$ to $40^{\circ}$. These experiments are discussed in Gomez-Rivas and Griera (2009, 2012) and only include models with composite plus intrinsic anisotropy.

(2) A series using pure OCLU-PLAST plasticine deformed at three different strain rates. These experiments are discussed in Gomez-Rivas and Griera (2011) and only include models with composite anisotropy.

(3) A series using different plasticine mixtures (of the trademarks OCLU-PLAST and JOVI) deformed them at the same strain rate. These experiments are discussed in Gomez-Rivas et al. (2015) and include models with either composite or composite plus intrinsic anisotropy.

These series of experiments were used to analyse fracture/ shear band formation and evolution in materials with different degrees and types of anisotropy deformed in dominant ductile conditions. Zheng et al. (2014) only considered the data that fit their MEM theory (our series 1), and ignored the two other series of experiments (2 and 3) where fracture formation angles do not fit the MEM fracture criterion.

The first problem of using the MEM criterion to explain experimental data is that it assumes that 
all fractures nucleate at $55^{\circ}$ from $\sigma_{1}$. However, the formation of shear fractures or shear bands in the experiments is much more complex than this. As explained in Gomez-Rivas (2008), Gomez- Rivas and Griera (2011, 2012) and Gomez-Rivas et al. (2015), shear fractures form by different mechanisms in the experiments: (a) nucleation from structures such as pinch-and-swell or boudinage (e.g., Schmalholz et al., 2008); (b) by collapse or propagation of one tension crack or void; (c) by propagation, collapse and coalescence of two or more tension cracks and/or voids (e.g., Bons et al., 2008), (d) by direct nucleation from local heterogeneities without the influence of pre-existing structures and (e) by linkage of pre-existing hybrid or shear fractures. All these mechanisms lead to the development of two macroscopically visible conjugate sets of shear fractures with progressive deformation.

Contrary to the theory by Zheng et al. (2011), initial shear fracture orientations with respect to the resolved $\sigma_{1}$ are highly variable depending on the considered parameters (Figure 1, Tables 1, 2, 3). It is true that the angle for our series (1) is on average $\sim 55^{\circ}$. There is, however, a significant spread in the data when individual fractures are considered, and the standard deviation of the mean is $\sim 7^{\circ}$ (Table 1). The initial orientations of shear fracture formation angles obtained from the series of experiments (2) and (3) clearly differ from the fixed $55^{\circ}$ predicted by the MEM criterion (Figure 1; Tables 2, 3). Average angles for the series (2) are close to $45^{\circ}$, while a wide range of orientations is observed in the series of experiments (3) using different plasticine mixtures series (ranging between $\sim 32^{\circ}$ and $\sim 55^{\circ}$, being $<45^{\circ}$ for most of the experiments).

The variability in initial angles of individual fractures is a consequence of the deformation conditions, material properties, heterogeneity of the anisotropic medium (degree and type of anisotropy) as well as the variable fracture formation mechanisms listed above, among other factors. This strong variability also happens in natural rocks and has been observed in many rock experiments as well. A variety of explanations for large angles with respect to maximum compression has been proposed in the literature, including the presence of pre-existing planar fabrics (Collettini et al., 2009; Fagereng et al., 2010), high fluid pressures and dissolution-precipitation creep (Fagereng et al., 2010), degree of anisotropy (Misra et al., 2009), large bulk deformation and fracture rotation towards the extension direction (Mancktelow, 2002; Gomez-Rivas et al., 2007; Scholz, 2007) or reactivation of pre-existing structures (Mancktelow and Pennacchioni, 2005; Pennacchioni and Mancktelow, 2007), among many other studies. Thus, finding a single criterion that can account for nucleation angles of all shear fractures in ductile anisotropic media is not straightforward.

\section{Some problems and contradictions of applying the MEM criterion to experiments}

We proposed in Gomez-Rivas and Griera (2012) that fracture nucleation angles depend on the angle of internal friction, the dilatancy angle as well as the orientation and degree of anisotropy. Additionally, we interpreted the large- fracture formation angles ( $>45^{\circ}$ on average) in Gomez-Rivas and Griera (2012) as a consequence of (a) the low propagation velocities of the existing fractures as a consequence of ductile deformation at their tips, which favours their simultaneous rotation while they slowly propagate, and (b) the influence of heterogeneities in the material that affect the orientation of the developed brittle fractures and shear bands. It is important to note that the models presented in Gomez- Rivas and Griera (2012) contained paper flakes statistically oriented parallel to layers, thus creating a strong intrinsic anisotropy that clearly affects macroscopic fracture orientations. On the contrary, shear fractures in the anisotropic experiments of Gomez-Rivas and 
Griera (2011), which did not contain paper flakes formed at an average angle of $45^{\circ}$ from $\sigma_{1}$, as predicted by Tresca or Von Mises criteria (e.g. Ramsay, 1980; Twiss and Moores, 1992; Pollard and Fletcher, 2005; Fagereng, 2013). Additionally, the results of the experiments published in Gomez-Rivas et al. (2015) demonstrate that the type of anisotropy also has a strong influence on fracture orientations. Particularly, only models with a combined composite-intrinsic anisotropy (see Griera et al., 2013) result in shear fractures or bands oriented on average at more than $45^{\circ}$ from 1 . Therefore, shear-fracture angles are thus clearly material-dependent.

Contrarily to the generally accepted principles of rock mechanics as well as to our experimental observations, the MEM criterion by Zheng et al. (2004, 2011, 2012) is supposed to be material-independent. An angle of $55^{\circ}$ between shear fractures and $\sigma_{1}$ is inferred as a universal value for ductile fractures for both homogeneous and heterogeneous media, as well as for both isotropic and anisotropic materials. Zheng et al. (2014) contradict themselves in their Figure 4 when they show how the resolved stress field deviates from the imposed deformation axes (i.e. what they call "deformation partition”). This graph shows that this deviation depends on the degree of anisotropy, so it is a material-dependent parameter. They state that our models are a case of " $100 \%$ deformation partitioning", which would mean for them that all deformation is accommodated by slip along the discrete fracture network. In our experiments, however, most of the deformation is distributed within the material, which deforms by dominant viscous flow and without loss of cohesion. They also propose that the MEM criterion is scale-independent, but it has been demonstrated that that the scale of anisotropy, and therefore its type, has a strong influence on the resulting deformation structures (e.g. see Griera et al., 2011, 2013).

Zheng and co-authors state that zones in between shear bands (or lozenges) are nearly strain-free areas. Their assumption that lozenges are strain-free areas and the use of the term "puzzles" assumes that the material behaves rigidly and that fracture orientations do not change. This is against the observations by many authors (e.g., Graham, 1980). Additionally, Zheng and co-authors assert that the MEM criterion assumes that the fracture angles remain constant with increasing deformation. Our three series of experiments illustrate how conjugate sets of shear fractures do rotate with progressive deformation, as is the case in most analysed natural cases. Moreover, conjugate sets of shear fractures are subjected to different amounts of normal and shear stress when anisotropy is oblique to the deformation axes. This implies that fractures have different chances for slip and propagation according to their orientation, thus determining their evolution. Slip along fractures of one set can potentially be locked depending on their angle with regard to $\sigma_{1}$ (e.g., Scholz, 2007; Gomez-Rivas and Griera, 2011; Gomez-Rivas et al., 2015).

\section{Conclusions}

The data of the three series of experiments presented in Gomez-Rivas and Griera (2012), and additional results disused in Gomez-Rivas and Griera (2011) and Gomez-Rivas et al. (2015), clearly indicate that both average and individual fracture formation angles do not fit with the MEM criterion of Zheng et al. (2004, 2011, 2012) and its associated implications. The similarity of average angles in the models of Gomez-Rivas and Griera (2012) is a spurious coincidence. Moreover, our three series of experiments demonstrate not only the angles at which fractures form, but also the type of structures (pinch-and-swell, boudinage, layer- parallel slip, tension cracks, voids, shear fractures/bands) as well as the statistical properties of fracture networks (density, connectivity and displacement/length relationship) strongly depend on the mechanical properties of the material, 
the degree and type of anisotropy and the conditions at which the material is deformed (applied strain rate and kinematic vorticity resulting from the orientation of the applied stress field). In conclusion, our experimental results should not be used to support the MEM criterion, since they clearly indicate that there is a strong material dependence on the angle at which shear fractures/bands nucleate in materials that undergo dominant ductile deformation.

\section{Acknowledgements}

We gratefully acknowledge A. Fagereng and an anonymous reviewer, whose suggestions have improved the manuscript.

\section{References}

Bons P D, Druguet E, Castaño L M, Elburg M A. 2008. Finding what is now not there anymore: Recognizing missing fluid and magma volumes. Geology, 36: 851-854

Collettinni C, Niejmeijer A, Viti C, Marone C. 2009. Fault zone fabrics and fault weakness. Nature, 462: 907-910

Fagereng A, Remitti F, Sibson R H. 2010. Shear veins observed within anisotropic fabric at high angles to the maximum compressive stress. Nat Geosci, 3: 482-485

Fagereng A. 2013. On stress and strain in a continuous-discontinuous shear zone undergoing simple shear and volume loss. J Struct Geol, 50: 44-53

Gomez-Rivas E, Bons P D, Griera A, Carreras J, Druguet E, Evans L. 2007. Strain and vorticity analysis using small-scale faults and associated drag folds. J Struct Geol, 29: 1882-1899

Gomez-Rivas E. 2008. Localización de deformación en medios dúctiles y anisótropos: estudio de campo, experimental y numérico. PhD thesis. Universitat Autònoma de Barcelona. http://hdl.handle.net/10803/3445

Gomez-Rivas E, Griera A. 2009. Influence of mechanical anisotropy on shear fracture development. Trabajos de Geología, 29: 305-311

Gomez-Rivas E, Griera A. 2011. Strain rate influence on fracture development in experimental ductile multilayers. Tectonophysics, 502: 345- 357

Gomez-Rivas E, Griera A. 2012. Shear fractures in anisotropic ductile materials: An experimental approach. J Struct Geol, 34: 61-76

Gomez-Rivas E, Griera A, Llorens M G. 2015. Fracturing of ductile anisotropic multilayers: Influence of material strength. Solid Earth, 6: 497-514

Graham R H. 1980. The role of shear belts in the structural evolution of the South Harris igneous complex. J Struct Geol, 2: 29-37

Griera A, Bons P D, Jessell M W, Lebensohn R A, Evans L, Gomez-Rivas E. 2011. Strain localization and porphyroclast rotation. Geology, 39: 275-278

Griera A, Llorens M G, Gomez-Rivas E, Bons P D, Jessell M W, Evans L A, Lebensohn R. 2013. Numerical modelling of porphyroclast and porphyroblast rotation in anisotropic rocks. Tectonophysics, 587: 4-29

Mancktelow N S. 2002. Finite-element modelling of shear zone development in viscoelastic materials and its implications for localisation of partial melting. J Struct Geol, 24: 1045-1053

Mancktelow N S, Pennacchioni G. 2005. The control of precursor brittle fracture and fluid-rock interaction on the development of single and paired ductile shear zones. J Struct Geol, 27: 645-661

Misra S, Mandal N, Chakraborty C. 2009. Formation of Riedel shear fractures in granular materials: Findings from analogue shear experiments and theoretical analyses. Tectonophysics, 471: 253-259

Pennacchioni G, Mancktelow N S. 2007. Nucleation and initial growth of a shear zone network within compositionally and structurally heterogeneous granitoids under amphibolite facies conditions. J Struct Geol, 29: 1757-1780

Pollard D D, Fletcher R C. 2005. Fundamentals of Structural Geology. Cambridge: Cambridge University Press

Ramsay J G. 1980. Shear zone geometry: A review. J Struct Geol, 2: 83-99

Schmalholz S M, Schmid D W, Fletcher R C. 2008. Evolution of pinch-and-swell structures in a power-law layer. J Struct Geol, 30: 649- 663

Scholz C H. 2007. Fault mechanics. In: Schubert G, ed. Treatise on Geophysics. Amsterdam: Elsevier. 441-483

Tong H. 2012. Comment and some questions on "Puzzles and the maximum effective moment (MEM) criterion in 
structural Geology”. J Struct Geol, 36: 81-84

Twiss R J, Moores E M. 1992. Structural Geology. New York: Freeman and Company

Zheng Y D, Wang T, Ma M, Davis G A. 2004. Maximum effective moment criterion and the origin of low-angle normal faults. J Struct Geol, 26: 271-285

Zheng Y D, Wang T, Zhang J J. 2011. Puzzles and the maximum effective moment (MEM) criterion in structural geology. J Struct Geol, 35: 1394-1405

Zheng Y D, Zhang J J, Wang T. 2012. Reply to comment and some questions on "Puzzles and the maximum-effective-moment (MEM) criterion in structural geology”. J Struct Geol, 36: 85-87

Zheng Y D, Zhang J J, Wang T. 2014. Interpretation of the experimental data provided by Gómez-Rivas and Griera (2012) in terms of the MEM-criterion. Sci China Earth Sci, 57: 2819-2824.
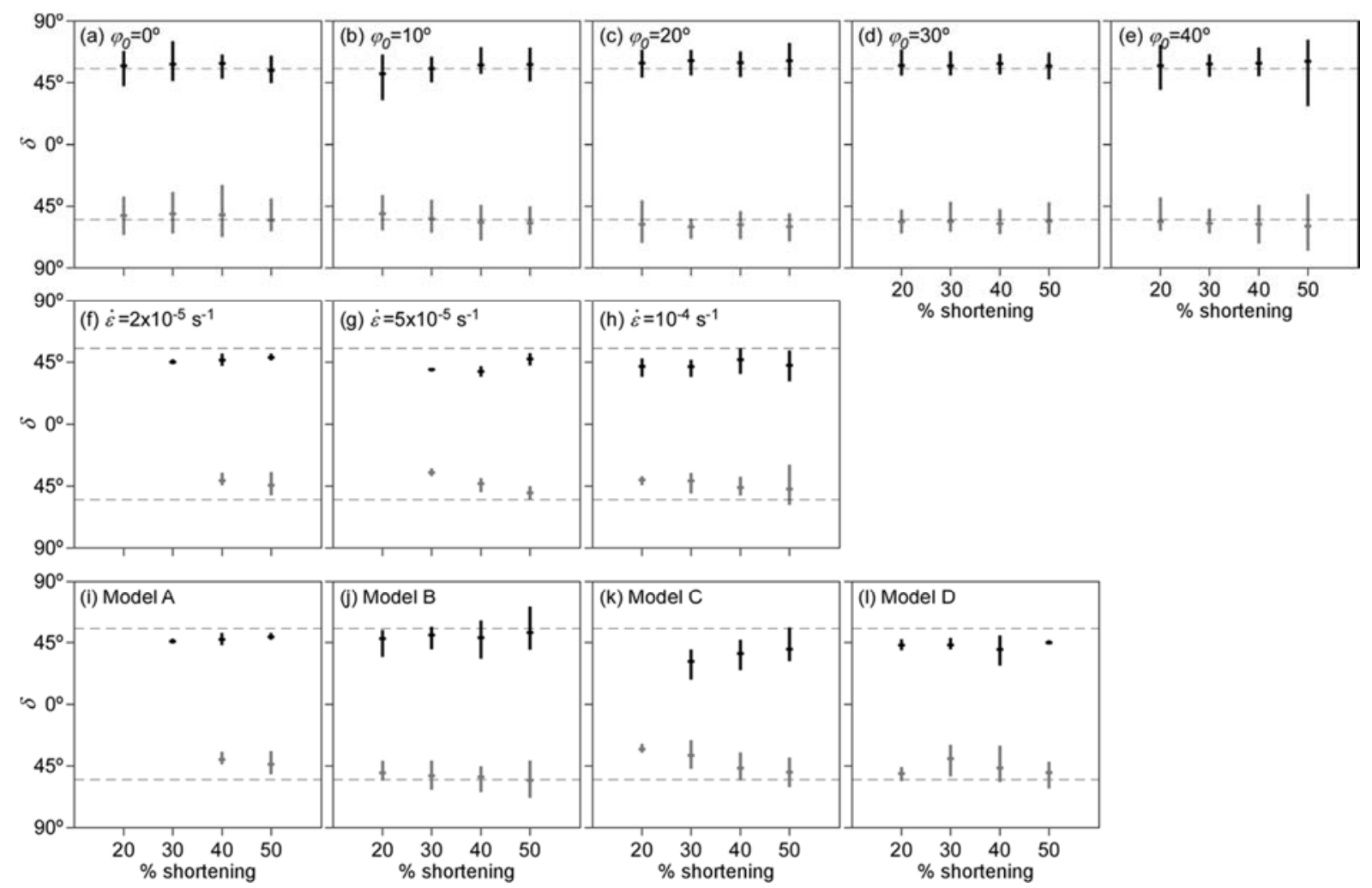

Figure 1. Candlestick graph showing the shear fracture nucleation orientations $(\delta)$ for all the experiments presented in the $\mathrm{PhD}$ thesis of Gomez-Rivas (2008), Gomez-Rivas and Griera $(2011,2012)$ and Gomez-Rivas et al. (2015). $\delta$-angles are measured with respect to the principal compressive stress. Graphs (a) to (e) present results of series 1, where the initial orientation of anisotropy with respect to the extension axis $\left(\varphi_{0}\right)$ was varied; (f) to (h) present results of series 2, where the applied strain rate was varied ( $\dot{\varepsilon}$ ); (i) to (l) present results of series 3 , where different plasticine mixtures were deformed. Candlesticks represent minimum, maximum and average orientations for each deformation stage (at intervals of $10 \%$ shortening) and each shear fracture set (black and grey represent sinistral and dextral sets, respectively). Values used to construct candlesticks are displayed in Tables 1, 2 and 3. Please note that for graphs (a) to (e) angles are calculated with respect to the resolved principal compression stress $\sigma_{1}{ }^{\prime}$, which has been taken as the obtuse bisector between average new fracture orientations at each stage. Dashed lines represent the orientation predicted by the MEM criterion (55). 
Table 1. Statistics of the orientations $(\delta$ ) of new shear fractures observed from experiments of series (1) (Gomez-Rivas, 2008; Gomez-Rivas and Griera, 2012). $\varphi_{0}$ is the initial orientation of anisotropy with respect to the extension axis. $\delta_{\text {st. dev }}$ accounts for the standard deviation. Please note that new shear fracture angles are calculated with respect to the resolved principal compression stress $\sigma_{1}$ ', which has been taken as the obtuse bisector between average new fracture orientations at each stage.

\begin{tabular}{|c|c|c|c|c|c|c|c|c|c|c|c|}
\hline \multirow{2}{*}{$\varphi_{0}$} & \multirow{2}{*}{ new fractures at } & \multicolumn{5}{|c|}{ Sinistral set of fractures } & \multicolumn{5}{|c|}{ Dextral set of fractures } \\
\hline & & $n$ & $\delta_{\text {average }}$ & $\delta_{\text {st.dev }}$ & $\delta_{\min }$ & $\delta_{\max }$ & $n$ & $\delta_{\text {average }}$ & $\delta_{\text {st.dev }}$ & $\delta_{\min }$ & $\delta_{\max }$ \\
\hline \multirow{4}{*}{$0^{\circ}$} & $20 \%$ sh. & 40 & 57.01 & 6.78 & 42.14 & 67.47 & 42 & 52.65 & 7.09 & 39.12 & 66.8 \\
\hline & $30 \%$ & 31 & 58.11 & 7.11 & 45.67 & 74.59 & 27 & 51.3 & 8.52 & 35.43 & 65.71 \\
\hline & $40 \%$ & 22 & 58.61 & 4.92 & 47.3 & 64.9 & 26 & 52.03 & 9.59 & 30.47 & 68.2 \\
\hline & $50 \%$ & 23 & 53.47 & 5.84 & 44.09 & 64.23 & 27 & 56.06 & 6.14 & 40.36 & 64.23 \\
\hline \multirow{4}{*}{$10^{\circ}$} & $20 \%$ & 37 & 51.17 & 7.06 & 31.94 & 64.85 & 31 & 51.17 & 7.39 & 37.78 & 63.31 \\
\hline & $30 \%$ & 24 & 54.83 & 5.59 & 44.61 & 63.12 & 22 & 54.83 & 6.28 & 41.38 & 65.01 \\
\hline & $40 \%$ & 22 & 57.54 & 4.57 & 50.94 & 70.41 & 18 & 57.54 & 7.15 & 45.11 & 70.99 \\
\hline & $50 \%$ & 23 & 57.91 & 6.2 & 45.33 & 69.95 & 25 & 57.91 & 5.76 & 46.23 & 66.61 \\
\hline \multirow{4}{*}{$20^{\circ}$} & $20 \%$ & 34 & 58.96 & 5.25 & 48.12 & 68.55 & 23 & 58.96 & 6.99 & 41.69 & 72.61 \\
\hline & $30 \%$ & 16 & 60.74 & 4.98 & 49.77 & 68.13 & 10 & 60.74 & 4.56 & 55.43 & 69.46 \\
\hline & $40 \%$ & 23 & 59.22 & 6.33 & 48.41 & 67.2 & 16 & 59.22 & 6.12 & 49.47 & 69.94 \\
\hline & $50 \%$ & 20 & 60.67 & 6.04 & 48.69 & 73.21 & 16 & 60.67 & 5.2 & 51.34 & 71.59 \\
\hline \multirow{3}{*}{$30^{\circ}$} & $20 \%$ & 24 & 57.11 & 4.66 & 49.68 & 68.63 & 19 & 57.11 & 5.03 & 48.45 & 65.76 \\
\hline & $30 \%$ & 17 & 56.97 & 5.3 & 49.94 & 67.18 & 8 & 56.97 & 6.91 & 42.62 & 64.6 \\
\hline & $50 \%$ & 23 & 56.72 & 5.18 & 46.88 & 66.26 & 18 & 56.72 & 7.39 & 43.06 & 66.29 \\
\hline \multirow{4}{*}{$40^{\circ}$} & $20 \%$ & 17 & 57 & 9.68 & 39.39 & 72.11 & 14 & 57 & 6.39 & 39.8 & 63.89 \\
\hline & $30 \%$ & 14 & 58.2 & 4.49 & 48.89 & 65.03 & 9 & 58.2 & 5.73 & 47.66 & 65.68 \\
\hline & $40 \%$ & 22 & 58.85 & 5.3 & 49.03 & 69.92 & 12 & 58.85 & 9.62 & 45.13 & 73.03 \\
\hline & $50 \%$ & 21 & 60.25 & 11.58 & 27.26 & 75.78 & 19 & 60.25 & 13.24 & 37.24 & 78.54 \\
\hline
\end{tabular}

Table 2. Statistics of the orientations ( $\delta$ ) of new shear fractures obtained from experiments of series (2) (Gomez-Rivas, 2008; Gomez-Rivas and Griera, 2011). $\dot{\varepsilon}$ is the strain rate applied by the deformation apparatus. $\delta_{\text {st. dev }}$ accounts for the standard deviation.

\begin{tabular}{|c|c|c|c|c|c|c|c|c|c|c|c|}
\hline \multirow{2}{*}{$\dot{\varepsilon}\left(\mathrm{s}^{-1}\right)$} & \multirow{2}{*}{ new fractures at } & \multicolumn{5}{|c|}{ Sinistral set of fractures } & \multicolumn{5}{|c|}{ Dextral set of fractures } \\
\hline & & $n$ & $\delta_{\text {average }}$ & $\delta_{\text {st.dev }}$ & $\delta_{\text {min }}$ & $\delta_{\max }$ & $n$ & $\delta_{\text {average }}$ & $\delta_{\text {st.dev }}$ & $\delta_{\min }$ & $\delta_{\max }$ \\
\hline \multirow{4}{*}{$2 \times 10^{-5}$} & $20 \%$ sh. & - & - & - & - & - & - & - & - & - & - \\
\hline & $30 \%$ & 2 & 45.34 & 2.31 & 43.71 & 46.98 & - & - & - & - & - \\
\hline & $40 \%$ & 6 & 46.76 & 3.42 & 42.22 & 51.28 & 7 & 41.09 & 3.53 & 35.4 & 44.73 \\
\hline & $50 \%$ & 4 & 48.69 & 2.11 & 46.29 & 51.35 & 7 & 44.53 & 5.44 & 35.18 & 52.01 \\
\hline \multirow{3}{*}{$5 \times 10^{-5}$} & $30 \%$ & 3 & 39.97 & 1.38 & 38.38 & 40.83 & 2 & 35.25 & 3.89 & 32.5 & 38 \\
\hline & $40 \%$ & 4 & 38.44 & 3.1 & 34.7 & 42.14 & 8 & 43.31 & 2.98 & 39.61 & 49.48 \\
\hline & $50 \%$ & 16 & 47.61 & 3.04 & 42.7 & 51.69 & 13 & 49.99 & 2.62 & 45.3 & 54.92 \\
\hline \multirow{3}{*}{$10^{-4}$} & $20 \%$ & 8 & 41.74 & 5.44 & 34.2 & 47.55 & 5 & 40.78 & 3.28 & 38.05 & 44.71 \\
\hline & $30 \%$ & 15 & 41.62 & 3.55 & 34.1 & 46.53 & 15 & 41.4 & 4.05 & 35.75 & 50.75 \\
\hline & $40 \%$ & 17 & 46.54 & 4.48 & 36.27 & 54.95 & 18 & 46.2 & 3.21 & 38.61 & 52.01 \\
\hline
\end{tabular}


Table 3. Statistics of the orientations $(\delta)$ of new shear fractures obtained from experiments of series (3)

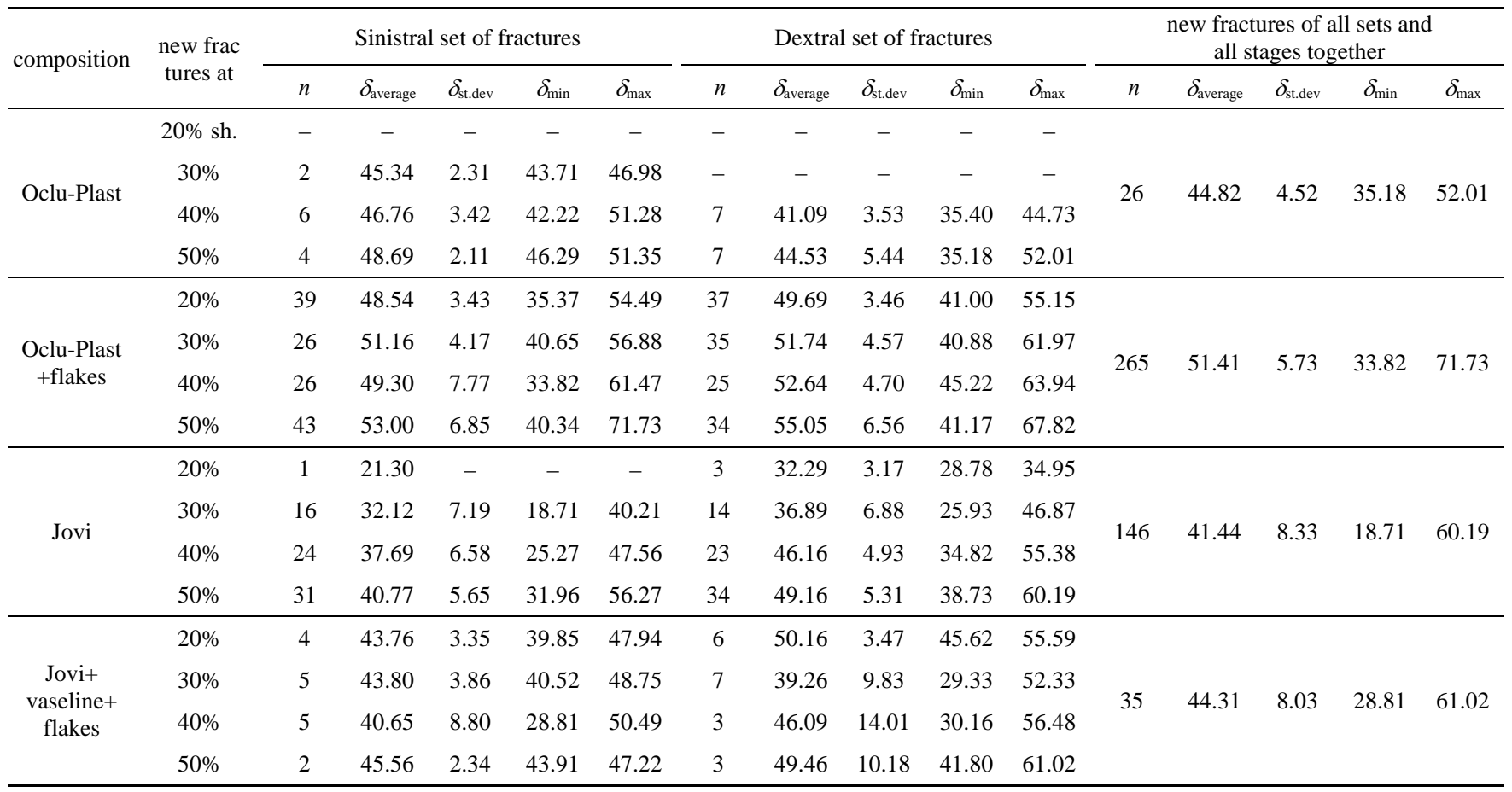

$\delta_{\text {st. dev }}$ accounts for the standard deviation and comp. for composition of the material. OCLU-PLAST and JOVI are the two plasticine manufacturers for each kind of plasticine. From Gomez-Rivas (2008) and Gomez-Rivas et al. (2015). 